Article

\title{
Vertically-Oriented $W_{2} S_{2}$ Nanosheets with a Few Layers and Its Raman Enhancements
}

\author{
Yukyung Shin ${ }^{1,+}$, Jayeong Kim ${ }^{2,+}\left(\mathbb{D}\right.$, Yujin Jang ${ }^{2}$, Eunji Ko ${ }^{2}$, Nam-Suk Lee ${ }^{3}$, \\ Seokhyun Yoon $2, *$ (1) and Myung Hwa Kim ${ }^{1, *}$ \\ 1 Department of Chemistry \& Nanoscience, Ewha Womans University, Seoul 03760, Korea; shinyk@ewhain.net \\ 2 Department of Physics, Ewha Womans University, Seoul 03760, Korea; jayeongkim@ewhain.net (J.K.); \\ yujin97@ewhain.net (Y.J.); ellenko94@ewhain.net (E.K.) \\ 3 National Institute for Nanomaterials Technology (NINT), Pohang University of Science and \\ Technology (POSTECH), Pohang 37673, Korea; nslee@postech.ac.kr \\ * Correspondence: syoon@ewha.ac.kr (S.Y.); myungkim@ewha.ac.kr (M.H.K.); \\ Tel.: +82-2-3277-4452 (S.Y.); +82-2-3277-4131 (M.H.K.) \\ + Authors equally contribute to this work.
}

Received: 18 August 2020; Accepted: 12 September 2020; Published: 16 September 2020

\begin{abstract}
Vertically-oriented two-dimensional (2D) tungsten disulfide $\left(\mathrm{WS}_{2}\right)$ nanosheets were successfully grown on a Si substrate at a temperature range between and $550{ }^{\circ} \mathrm{C}$ via the direct chemical reaction between $\mathrm{WCl}_{6}$ and $\mathrm{S}$ in the gas phase. The growth process was carefully optimized by adjusting temperature, the locations of reactants and substrate, and carrier gas flow. Additionally, vertically-oriented $2 \mathrm{D} \mathrm{WS}$ nanosheets with a few layers were tested as a surface-enhanced Raman scattering substrate for detecting rhodamine 6G (R6G) molecules where enhancement occurs from chemical enhancement by charge transfer transition from semiconductor). Raman spectra of R6G molecules adsorbed on vertically-oriented $2 \mathrm{D} \mathrm{WS}$, nanosheets exhibited strong Raman enhancement effects up to 9.2 times greater than that on the exfoliated $\mathrm{WS}_{2}$ monolayer flake sample. From our results, we suggest that the $\mathrm{WS}_{2}$ nanosheets can be an effective surface-enhanced Raman scattering substrate for detecting target molecules.
\end{abstract}

Keywords: tungsten disulfide; nanosheet; chemical vapor deposition; surface-enhanced Raman scattering

\section{Introduction}

Considerable attention has been devoted to two-dimensional (2D) materials as promising functional materials, in particular to transition metal dichalcogenides (TMDCs) [1-4], due to rapid advances in synthesis, transfer, spectroscopic detection, and manipulation. Since 2D TMDCs have unique physicochemical properties such as high mobility, large surface area, and significant catalytic activities, they can be effectively used for efficient light harvesting, sensitive photo-detection, and catalytic conversion systems [5-8]. Among 2D TMDCs, particularly, tungsten disulfide $\left(\mathrm{WS}_{2}\right)$, with direct optical band gaps of 1.35 and $2.05 \mathrm{eV}$ for bulk and monolayer structure, is of great interest due to its particular semiconducting behavior, intrinsic electrical conductivity, and electrocatalytic property when the number of layers is lower [9-11]. Recent work shows that 2D WS 2 nanostructures could exhibit the enhancement of Raman signals for adsorbed molecules as a function of the number of layers [12]. Although a variety of synthetic methodologies were thus suggested to grow single and few-layer nanosheets of the high-quality $2 \mathrm{D} \mathrm{WS}$, the synthesis and characterization of vertically-oriented $2 \mathrm{D}$ $\mathrm{WS}_{2}$ nanosheets have rarely been reported [13-16].

In this work, we described a novel growth process and its characterizations of vertically-oriented $2 \mathrm{D} \mathrm{WS} \mathrm{W}_{2}$ nanosheets using chemical vapor deposition (CVD). Although Li et al. previously reported 
$\mathrm{MoS}_{2}$ and $\mathrm{WS}_{2}$ inorganic fullerene-like nanostructures and nanoflowers with a similar growth method using atmospheric pressure chemical vapor deposition (APCVD) [17], our growth process makes it possible to obtain vertically-oriented $2 \mathrm{D} \mathrm{WS} \mathrm{W}_{2}$ nanosheets with a few layers on a Si substrate by carefully controlling the gas flow and the different location of the substrate at a much lower temperature. Thus, this synthetic methodology could offer an efficient growth process for 2D materials with high density. To utilize our structure, we performed surface-enhanced Raman scattering (SERS) experiments for rhodamine $6 \mathrm{G}(\mathrm{R} 6 \mathrm{G})$ molecules adsorbed on vertically-oriented layers of $2 \mathrm{D} \mathrm{WS}_{2}$ nanosheets. It is well known that SERS could enhance inherently weak Raman scattering cross-sections by many orders of magnitude using specially-prepared substrates on which samples to be measured are adsorbed [18-21]. Most of SERS substrates consist of noble metals using surface plasmon resonance in the visible range; however, semiconducting substrates are also being used for decent enhancement, whose enhancement mechanism is mainly associated with charge transfer between the substrate and the adsorbate molecules [22-24]. Recently, 2D materials, including TMDC materials are also being used as SERS substrates [25-27].

\section{Materials and Methods}

To grow $2 \mathrm{D} \mathrm{WS}_{2}$ nanosheets, tungsten hexachloride $\left(\mathrm{WCl}_{6}\right.$, Sigma-Aldrich, Seoul, Korea) was placed in the quartz boat at the center of the quartz tube of $60 \mathrm{~cm}$ length with a 2.5-inch diameter in the furnace, as illustrated in Figure 1 [28]. Another quartz boat containing sulfur powder (Sigma-Aldrich, $99.98 \%$, Seoul, Korea) was then placed at $15 \mathrm{~cm}$ upstream from the quartz boat with $\mathrm{WCl}_{6}$, which was near the outside edge of the heat zone in the furnace. The $\mathrm{Si}(001)$ substrate at the growth region was placed face-down above the quartz boat with $\mathrm{WCl}_{6}$. The gap distance between $\mathrm{WCl}_{6}$ and the Si substrate was approximately $10 \mathrm{~mm}$. Before starting the growth process, the quartz tube was flushed with the carrier gas helium (He, 99.999\%, Dong-A Industrial Gas, Seoul, Korea) for $10 \mathrm{~min}$ at 400 standard cubic centimeter per minute (sccm). The furnace was gradually heated up to $450 \sim 550{ }^{\circ} \mathrm{C}$ at the ramping speed of $26^{\circ} \mathrm{C}$ per minute and maintained at the growth temperature for $20 \mathrm{~min}$. During the growth process, the temperature at the location of the sulfur powder was kept between 180 and $200^{\circ} \mathrm{C}$. For the whole process, helium (He) was continuously flowed at the flow rate of $10 \mathrm{sccm}$. After finishing the growth process, the furnace (Lindberg/Blue M, Thermo Fisher Scientific, Waltham, MA, USA) was turned off and gradually cooled down to room temperature.

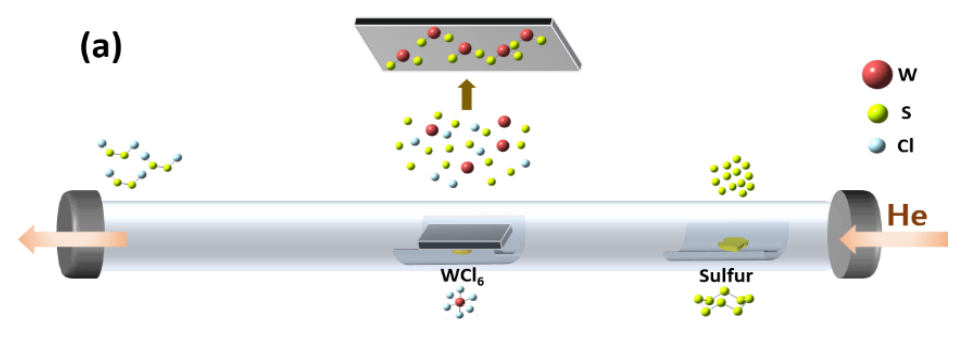

(b)

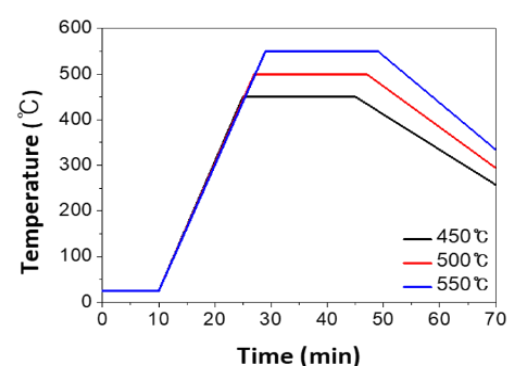

Figure 1. (a) Scheme of the chemical deposition process of the $\mathrm{WS}_{2}$ nanosheets and (b) temperature profile during the growth process. 
The characteristics of $2 \mathrm{D} \mathrm{WS}_{2}$ nanosheets on a Si substrate were analyzed by scanning electron microscopy (FE-SEM, JEOL JSM-6700F, Tokyo, Japan), high-resolution transmission electron microscopy (HR-TEM, with a probe Cs-corrector, JEM-2100F, Tokyo, Japan), X-ray diffraction (XRD, Rigaku diffractometer, Tokyo, Japan with Ni filtered $\mathrm{Cu}-\mathrm{K} \alpha$ radiation, $\lambda=0.15418 \mathrm{~nm}$ ), X-ray photoelectron spectroscopy (XPS with a Theta probe AR system and X-ray source with monochromated $\mathrm{Al} \mathrm{K} \alpha, \mathrm{h} v=1486.6 \mathrm{eV}$, JEOL, Tokyo, Japan), and Raman spectroscopy (Horiba, Kyoto, Japan). The atomic ratios of W/S in the form of nanosheets were carefully measured by energy-dispersive $\mathrm{X}$-ray spectroscopy (EDX).

For Raman measurements, $5 \times 10^{-4} \mathrm{M}$ R6G solution was prepared by adding $500 \mu \mathrm{L}$ of ethanol to $500 \mu \mathrm{L}$ of $10^{-3} \mathrm{M}$ R6G aqueous solution for effective drying. We drop-casted $12.5 \mu \mathrm{L}$ of R6G solution on $2 \mathrm{D} \mathrm{WS} \mathrm{W}_{2}$ nanosheets and exfoliated $\mathrm{WS}_{2}$ monolayer flake samples and waited for natural drying. Raman spectra of R6G molecules adsorbed on $\mathrm{WS}_{2}$ samples were measured by using a McPherson 207 spectrometer equipped with a nitrogen-cooled charge-coupled-device (CCD) array detector at room temperature. The samples were excited with a $532 \mathrm{~nm}(2.33 \mathrm{eV})$ diode-pumped solid state (DPSS) laser, focused to $\sim 1 \mu \mathrm{m}$ diameter spot using a microscope objective $(100 \times)$. The excitation power was less than $0.05 \mathrm{~mW}$ to avoid laser heating and the exposure time was $60 \mathrm{~s}$.

To estimate local electric field response distribution near the $\mathrm{WS}_{2}$ surface, finite-difference time-domain (FDTD) calculations (Lumerical Inc., Vancouver, Canada) were carried out. As the size of each bundle of nanosheets is a few $\mu \mathrm{m}$ and the size of the laser beam used in the Raman experiment is about $1 \mu \mathrm{m}$, we built the flower-like structure by making a board that was $1 \times 0.01 \times 0.4 \mu \mathrm{m}$ and rotating it around a vertical (z) axis so that it has 16 leaves. A plane-wave source was used and the boundary conditions for the periodic structure were configured: anti-symmetric for $\times$ boundaries, symmetric for $\mathrm{y}$ boundaries, and perfectly matched layer (PML) for $\mathrm{z}$ boundaries. Due to the thickness of single layer R6G molecules, we set the maximum mesh size over the nanoflower structure as $0.3-1 \mathrm{~nm}$.

\section{Results and Discussion}

Figure 2 shows SEM images of $2 \mathrm{D} \mathrm{WS}$ nanosheets grown at 450,500 , and $550{ }^{\circ} \mathrm{C}$ on a Si substrate. In SEM images, the morphology of the thin-layered $\mathrm{WS}_{2}$ nanostructures clearly represents a distinct flower-like nanostructure by standing on the vertical direction against the substrate. The density of vertically-oriented $2 \mathrm{D} \mathrm{WS} \mathrm{W}_{2}$ nanostructures gradually decreased likely because $2 \mathrm{D} \mathrm{WS}$ nanosheets with larger sizes formed due to the faster growth rate at higher temperature. In Figure $2 \mathrm{~b}, \mathrm{~d}, \mathrm{f}$, the high magnification SEM images indicate that the lateral size of the 2D WS 2 nanosheets is approximately $2 \mu \mathrm{m}$ and their thickness is less than $10 \mathrm{~nm}$. This kind of asymmetric crystal growth in the out-of-plane might be responsible for the weak interlayer coupling between $\mathrm{WS}_{2}$ crystal structures. Particularly, the favorable vertical orientation of the $2 \mathrm{D} \mathrm{WS}{ }_{2}$ nanosheets might be attributed to the compression and extrusion between $\mathrm{WS}_{2}$ island layers initially formed on the $\mathrm{Si}$ substrate [16]. In addition, the face-down position of the $\mathrm{Si}$ substrate with respect to the $\mathrm{WCl}_{6}$ source would be beneficial to obtaining a high flux of supersaturated $\mathrm{WS}_{2}$ vapors into the Si substrate, resulting in the vertical growth of $\mathrm{WS}_{2}$ nanosheets [16,27].

Figure 3 shows the XRD pattern of the $2 \mathrm{D} \mathrm{WS}$ nanosheets grown at different temperatures, indicating that there are only four prominent peaks at $14.6^{\circ}, 29.1^{\circ}, 44.2^{\circ}$, and $60.2^{\circ}$, which can be attributed to (002), (004), (006), and (008) planes of the hexagonal phase (JCPDS card No: 08-0237), respectively [29]. Whereas the $\mathrm{XRD}$ pattern at $550^{\circ} \mathrm{C}$ illustrates the pure hexagonal $\mathrm{WS}_{2}$ crystalline phase, the XRD peaks between $23^{\circ}$ and $25^{\circ}$ at $450{ }^{\circ} \mathrm{C}$ and $500^{\circ} \mathrm{C}$ are associated with $\mathrm{WO}_{3}$ impurity. Interestingly, the distinct appearance of only $(00 l)$ reflections represents the characteristic of the specific orientation of the $2 \mathrm{D} \mathrm{WS} 2$ crystal structure along the $c$ axis direction. Based on the peak at $14.6^{\circ}$ assigned as the (002) plane, the lattice constant of $c$ axis is estimated as $12.22 \AA$. Additionally the intense sharp peaks at $550{ }^{\circ} \mathrm{C}$ confirm the highly crystalline nature of $2 \mathrm{D} \mathrm{WS}$ layered structures. 


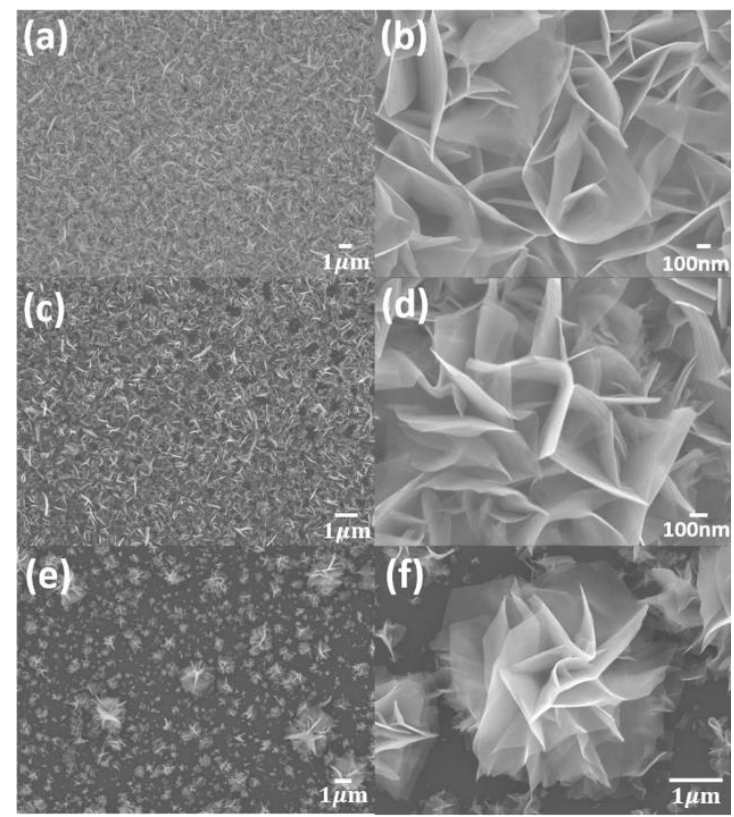

Figure 2. SEM images of vertically-oriented $\mathrm{WS}_{2}$ nanosheets grown on a Si substrate at $(\mathbf{a}, \mathbf{b}) 450$, (c,d) 500 , and $(\mathbf{e}, \mathbf{f}) 550{ }^{\circ} \mathrm{C}$.

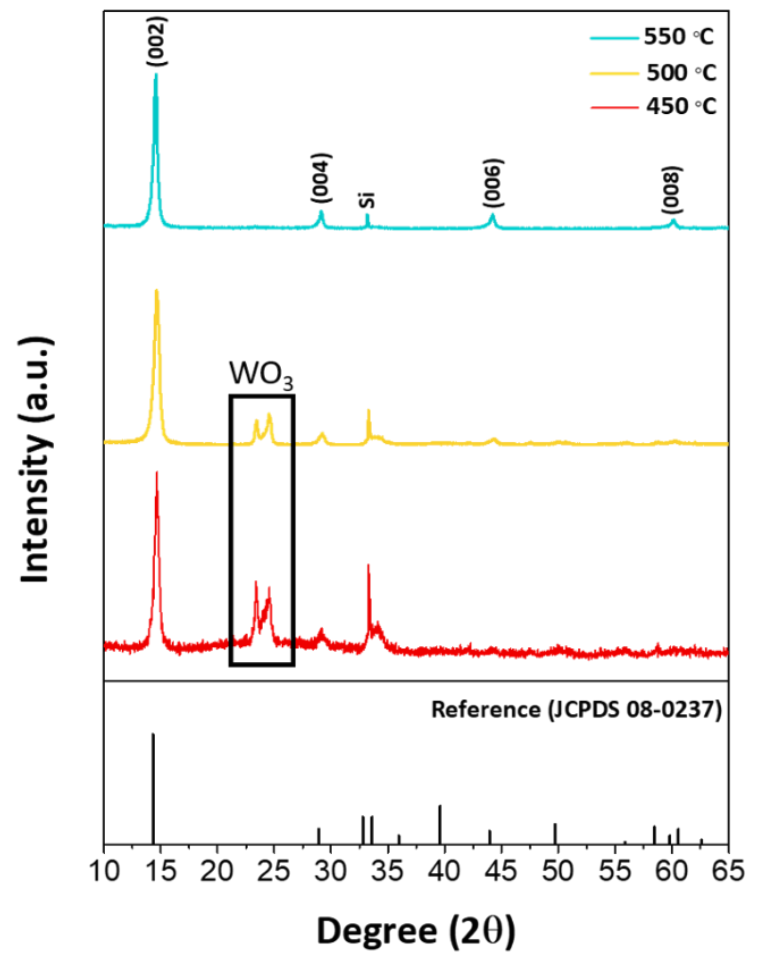

Figure 3. XRD patterns of vertically oriented $\mathrm{WS}_{2}$ nanosheets grown on a Si substrate at 450,500 and $550{ }^{\circ} \mathrm{C}$.

In the TEM measurement, the formation of different numbers of layered $\mathrm{WS}_{2}$ nanosheets is clearly identified by the relative difference in brightness with distinct edges as shown in Figure $4 \mathrm{a}$. The fast Fourier transform (FFT) image in Figure $4 \mathrm{~b}$ inset also confirms the existence of specific crystalline planes, corresponding to the $\mathrm{WS}_{2}$ crystal structures, indicating the highly single-crystalline nature of the nanosheet. Energy-dispersive X-ray spectroscopy (EDS) elemental mapping analysis from the Z-contrast high-angle annular dark-field scanning transmission electron microscopy (HAADF-STEM) 
image exhibits that $\mathrm{W}$ and $\mathrm{S}$ atoms are homogeneously distributed to the entire nanosheet, resulting in the almost stoichiometric atomic ratio of 1:1.97.
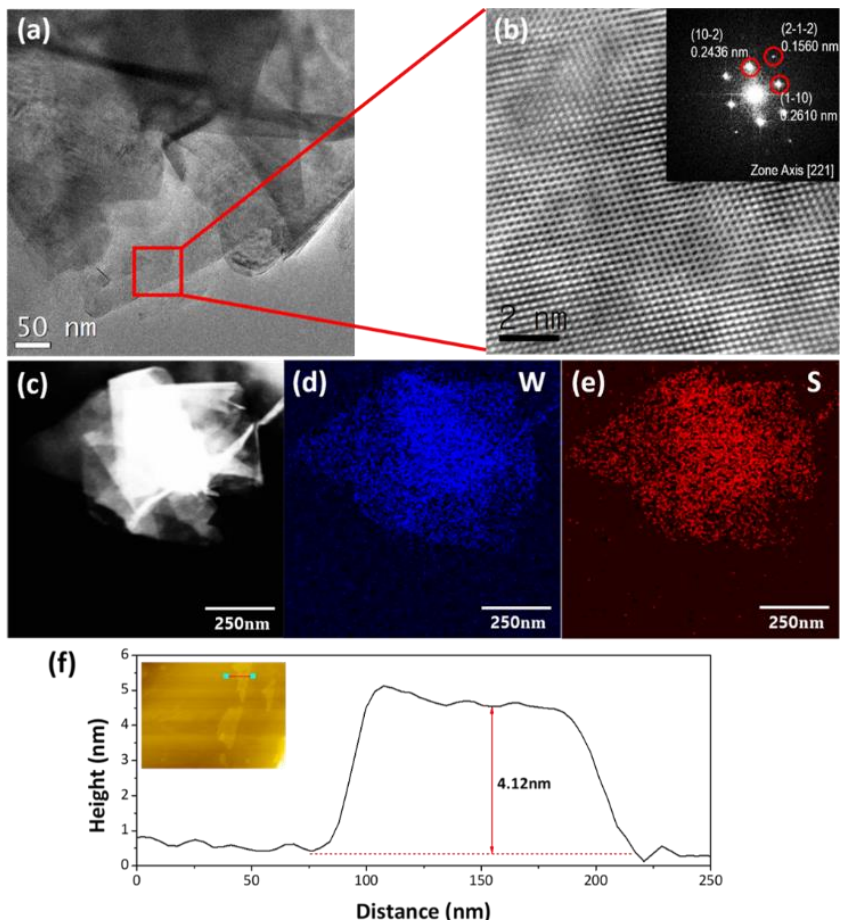

Figure 4. (a) Low-magnification TEM image of $\mathrm{WS}_{2}$ nanosheets at $550{ }^{\circ} \mathrm{C}$, (b) high-resolution TEM image and the fast Fourier transform (FFT) pattern of $\mathrm{WS}_{2}$ nanosheets(inset) at $550{ }^{\circ} \mathrm{C},(\mathbf{c}-\mathbf{e})$ EDS elemental mapping analysis of $\mathrm{W}$ and $\mathrm{S}$ atoms in $\mathrm{WS}_{2}$ nanosheets, (f) the height profile of $\mathrm{WS}_{2}$ nanosheets grown at $550{ }^{\circ} \mathrm{C}$ by atomic force microscopy (AFM) line scan.

The atomic force microscopy (AFM) measurement in Figure $4 \mathrm{f}$ depicts that the height of a single $\mathrm{WS}_{2}$ nanosheet is about $4.12 \mathrm{~nm}$, which corresponds to about five layers of the $\mathrm{WS}_{2}$ nanosheets.

Figure 5 represents the XPS spectra for the $\mathrm{WS}_{2}$ nanosheets, which could provide information regarding the surface composition and the chemical states of the elements in nanostructures. XPS spectra of $\mathrm{WS}_{2}$ nanosheets grown at $550{ }^{\circ} \mathrm{C}$ indicates that while the binding energies of 33.08 and $35.0 \mathrm{eV}$ are associated with the $\mathrm{W} 4 \mathrm{f}_{7 / 2}$ and $\mathrm{W} 4 \mathrm{f}_{5 / 2}$ core level peaks, two main peaks at 162.7 and $163.9 \mathrm{eV}$ correspond to $S 2 p_{3 / 2}$ and $2 p_{1 / 2}$ of the divalent ion $\left(S^{2-}\right)$ state, respectively [30]. These features are closely consistent with the XPS spectra for the exfoliated bulk $\mathrm{WS}_{2}$ structure.
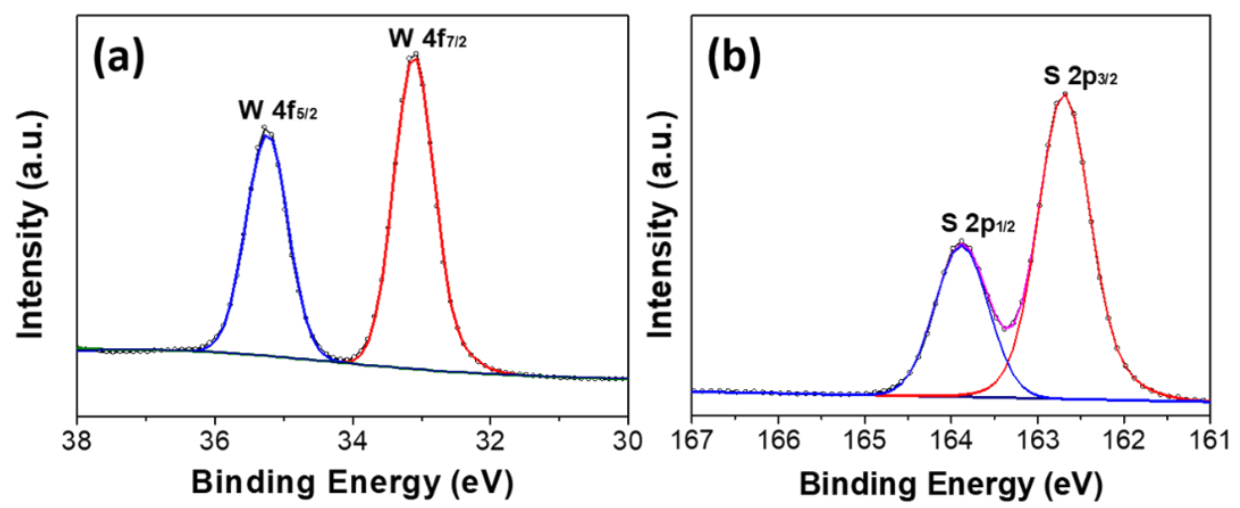

Figure 5. X-ray photoelectron spectroscopy (XPS) spectra of two-dimensional (2D) $\mathrm{WS}_{2}$ nanosheets at $550^{\circ} \mathrm{C}$ and (a) W $4 \mathrm{f}$ and (b) S 2 p regions. 
In our study, the growth of the $\mathrm{WS}_{2}$ nanosheets could be interpreted by the direct chemical reaction between $\mathrm{WCl}_{6}$ and $\mathrm{S}$ atoms in the gas phase on a Si substrate under the flow of the carrier gas (He) as given in Equation (1) [17]:

$$
\mathrm{WCl}_{6}+8 \mathrm{~S} \rightarrow \mathrm{WS}_{2}+3 \mathrm{~S}_{2} \mathrm{Cl}_{2}
$$

It is suggested that the reaction between $\mathrm{WCl}_{6}$ and $\mathrm{S}$ atoms initially forms tiny $\mathrm{WS}_{2}$ nuclei at the growth temperature. The continuous feeding of $\mathrm{WS}_{2}$ into the nucleation sites can then lead to the two-dimensional layered structure of $\mathrm{WS}_{2}$ as a favorable form. In addition, the use of excessive $S$ powder is beneficial for completely changing the chlorides to $\mathrm{WS}_{2}$. Generally, since the reaction in Equation (1) is highly exothermic at elevated temperatures, it leads to a rapid reaction progression with a high degree of supersaturation of the vapors and, hence, to fast nucleation. Thus, we expected that numerous nuclei of $\mathrm{WS}_{2}$ could be initially formed in the vapor phase at the near heating zone. It is likely that some nuclei grow larger relative to others and subsequently precipitate on the substrates, which are placed at the upper position from the $\mathrm{WCl}_{6}$ precursors. The deposited $\mathrm{WS}_{2}$ nanostructures then play a crucial role as the efficient growth template and promote the subsequent vertical growth of $\mathrm{WS}_{2}$ nanosheets due to the high strain energy by the compression between $\mathrm{WS}_{2}$ island layers as previously proposed [16]. During the growth process at high temperature, additionally, the gravity force might affect the vertical orientation of layered structures with relatively large radii of curvature and thin walls from deposited $\mathrm{WS}_{2}$ nuclei on a Si substrate, resulting in flower-like nanostructures. Although the whole reaction system was tightly sealed and protected by a flow of $\mathrm{He}$, traces of oxygen could cause the oxidation of the products. As shown in Figure 3, the minor product, $\mathrm{WO}_{3}$, was inevitable at the growth temperature of 450 and $500{ }^{\circ} \mathrm{C}$, whereas it was completely absent at $550{ }^{\circ} \mathrm{C}$. Therefore, this suggested that the growth temperature plays a critical role in efficiently obtaining the $\mathrm{WS}_{2}$ nanosheets with high crystallinity and high density on a Si substrate due to different thermodynamics and kinetics for the growth behavior.

Figure 6a represents the Raman spectra of $2 \mathrm{D} \mathrm{WS}_{2}$ nanosheets substrates grown at different growth temperatures and an exfoliated flake sample. All the nanosheet samples were of high quality as they all showed characteristic $W_{2}$ phonon peaks at $532 \mathrm{~nm}$ excitation as an exfoliated flake sample. Figure $6 \mathrm{~b}$ shows the topography and line profile of the exfoliated flake sample from which the flake is seen to be monolayer-thick. Note that the intensity of $A_{1 g}(\Gamma)$ peak at $\sim 420 \mathrm{~cm}^{-1}$ in nanosheet samples is much stronger. Suggesting that the nanosheet samples were much thicker than the exfoliated flake sample, which is also confirmed from Figure 1 where the thicknesses of the nanosheets are measured as $\sim 10 \mathrm{~nm}$.
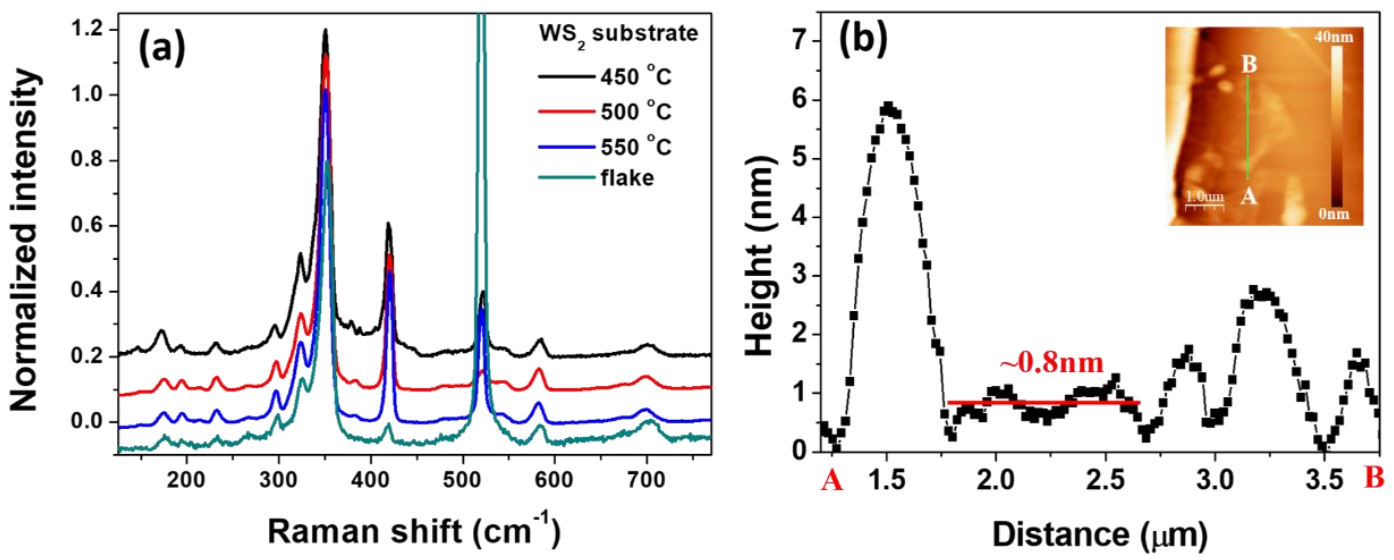

Figure 6. (a) Raman spectra of $2 \mathrm{D} \mathrm{WS}_{2}$ nanosheets substrates and monolayer $\mathrm{WS}_{2}$ flake substrate. Spectra are offset for clarity. (b) AFM topography line profile results of a $\mathrm{WS}_{2}$ flake sample, showing the $\mathrm{WS}_{2}$ flake sample is monolayer $\mathrm{WS}_{2}$. 
Figure 7 shows the Raman spectra of R6G molecules deposited on the 2D WS 2 nanosheets grown at various temperatures and an exfoliated $\mathrm{WS}_{2}$ monolayer flake. In Figure $7 \mathrm{a}$, vibration modes of adsorbed R6G molecules are clearly shown at 612, 770, 1181, 1303, 1361, 1500, 1570, and $1650 \mathrm{~cm}^{-1}$ from all the $2 \mathrm{D} \mathrm{WS}_{2}$ substrates. The strongest intensity of R6G molecular vibrations was observed in the 2D $\mathrm{WS}_{2}$ nanosheets grown at $450{ }^{\circ} \mathrm{C}$ and the intensity was weaker for nanosheets with higher growth temperatures. Since we did not observe any R6G Raman signal from solution with R6G concentration of $5 \times 10^{-4} \mathrm{M}$ and we only observed R6G vibration peaks when we drop-casted the R6G solution on $\mathrm{WS}_{2}$ substrates, the observation of R6G Raman responses is a direct result of surface-enhanced Raman scattering (SERS).
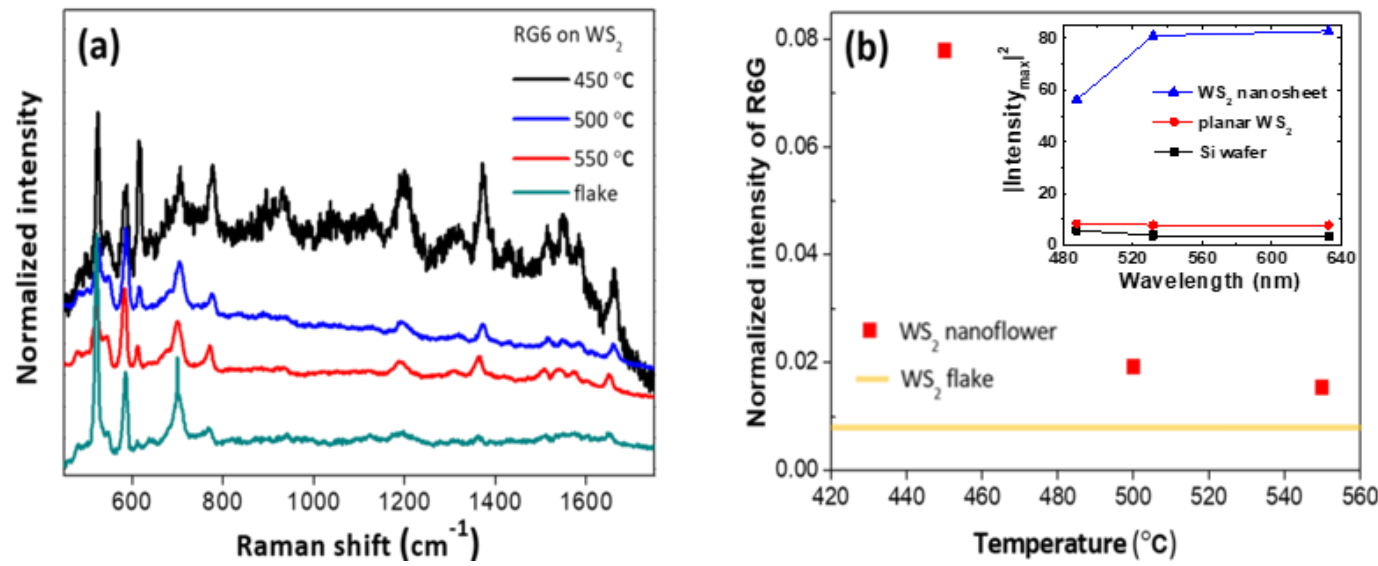

Figure 7. (a) Raman spectra of R6G molecules adsorbed on $\mathrm{WS}_{2}$ nanosheets substrates grown at 450 , 500 , and $550^{\circ} \mathrm{C}$, and an exfoliated $\mathrm{WS}_{2}$ monolayer flake sample. Spectra are offset for clarity. Intensities are normalized to the intensity of $\mathrm{WS}_{2} \mathrm{E}^{1}{ }_{2 \mathrm{~g}}$ mode. (b) Normalized (to $\mathrm{WS}_{2} \mathrm{E}^{1}{ }_{2 \mathrm{~g}}$ mode) intensity of R6G $1361 \mathrm{~cm}^{-1}$ mode. Filled squares represent the normalized intensities observed from $\mathrm{WS}_{2}$ nanoflower (nanosheet) samples, and the straight line represents the normalized intensity observed from an exfoliated $\mathrm{WS}_{2}$ monolayer flake. Inset: Finite-difference time-domain (FDTD) calculation results of scattered electric field intensity squared as a function of incident wavelength. Note that scattered intensities from both $\mathrm{WS}_{2}$ samples are larger than that from $\mathrm{Si}$ wafer confirming that it is from surface-enhanced Raman scattering (SERS) effects.

Since $\mathrm{WS}_{2}$ is a semiconductor, its surface plasmon energy is far from the visible range and the enhancement is entirely due to chemical, or charge transfer, enhancement. In our case, with visible $(2.33 \mathrm{eV})$ excitation, charge transfer can only occur from the valance band (VB) of $\mathrm{WS}_{2}$ to the lowest unoccupied molecular orbital (LUMO) level of R6G. The largest Raman enhancement thus occurs when the energy difference between VB and LUMO matches the excitation energy of the incident laser [31,32]. The samples grown at different temperatures have different thicknesses and different impurity contents, leading to different positions of each energy level. As a result, the relevant energy difference between VB and LUMO is different sample by sample and it is likely that the $450{ }^{\circ} \mathrm{C}$ sample has an energy difference close to the laser excitation energy.

Another factor that can affect the enhancement is the difference in the surface area among samples, which is directly related to the amount of charge transfer between the substrate and the sample. However, this was less likely in our case because the diameter of the focused laser beam was about $1 \mu \mathrm{m}$, which is larger, or at least comparable, to each flower structure, so the difference between the surface area from different samples was not significant. Although the intensity of R6G molecules from vertically-oriented $2 \mathrm{D} \mathrm{WS}$ nanosheets grown at $550{ }^{\circ} \mathrm{C}$ was the lowest among the three samples, it was still more than twice as strong as the measured intensity from an exfoliated flake sample. The Raman response in vertically-oriented $\mathrm{WS}_{2}$ nanosheets was estimated to be up to 9.2 times greater than in the monolayer flake sample as shown in Figure $7 \mathrm{~b}$. This can be explained by the difference in surface area, which is much larger in nanosheets. As can be seen in the Figure $7 \mathrm{~b}$ inset, the FDTD calculation also 
confirms that the scattered intensity from a nanosheet sample was always larger than that from a planar sample. The difference depends on the incident light wavelength and the intensity was 4.6 times larger in a nanosheet sample than that in a planar sample for $532 \mathrm{~nm}$ excitation, which is consistent with the experimental results. Consequently, this suggests that vertically-oriented $2 \mathrm{D} \mathrm{WS}_{2}$ nanosheets are much more efficient than exfoliated flakes as SERS substrates. Note that since the main cause of Raman enhancement in our case was not surface plasmon resonance but the photo-induced charge transfer between molecules and substrates, the FDTD results should be taken as an indicator as to how structure/morphology affect the local electric field response in our case.

\section{Conclusions}

We successfully introduced a novel synthesis of vertically-oriented $2 \mathrm{D} \mathrm{WS}_{2}$ nanosheets with a few layers in high density via chemical vapor deposition using $\mathrm{WCl}_{6}$ as the $\mathrm{W}$ precursor and sulfur by carefully controlling the gas flow and the location of the substrate at low temperature. Raman spectra of R6G molecules adsorbed on vertically-oriented 2D WS 2 nanosheets exhibited strong Raman enhancement effects compared to the monolayer flake of $2 \mathrm{D} \mathrm{WS}_{2}$. This suggested that the charge transfer transition from semiconductor substrates to molecular LUMO may be attributed to an efficient or a dominant process for attaining a large Raman enhancement.

Author Contributions: M.H.K. and S.Y. conceived the idea, performed the analysis, and wrote the original manuscript; conceptualization, M.H.K., and S.Y.; methodology, Y.S. and J.K.; validation, Y.J. and N.-S.L.; formal analysis, Y.S., J.K., and Y.J.; data curation, Y.S., J.K, Y.J., and E.K.; writing-original draft preparation, M.H.K., S.Y. and Y.S.; writing—review and editing, M.H.K. and S.Y.; visualization, Y.S., J.K., E.K. and N.-S.L.; supervision M.H.K. and S.Y.; project administration, M.H.K., and S.Y.; funding acquisition, M.H.K., and S.Y. All authors have read and agreed to the published version of the manuscript.

Funding: This research was supported by the National Research Foundation of Korea (NRF) Grant funded by the Korean Government (2016R1D1A1B01009032, 2018R1A6A1A03025340 for S.Y. and 2019R1F1A1062799 for M.H.K.).

Acknowledgments: We acknowledge Juran Kim and William Jo for helpful AFM measurement.

Conflicts of Interest: The authors declare no conflict of interest.

\section{References}

1. Wang, Q.H.; Kalantar-Zadeh, K.; Kis, A.; Coleman, J.N.; Strano, M.S. Electronics and optoelectronics of two-dimensional transition metal dichalcogenides. Nat. Nanotechnol. 2012, 7, 699-712. [CrossRef]

2. Yang, S.; Zhang, P.P.; Nia, A.S.; Feng, S.L. Emerging 2D materials produced via electrochemistry. Adv. Mater. 2020, 32, 1907857. [CrossRef]

3. Yun, Q.B.; Li, L.X.; Hu, Z.N.; Lu, Q.P.; Chen, B.; Zhang, H. Layered transition metal dichalcogenide-based nanomaterials for electrochemical energy storage. Adv. Mater. 2020, 32, 1903826. [CrossRef]

4. Karunakaran, S.; Pandit, S.; Basu, B.; De, M. Simultaneous exfoliation and functionalization of $2 \mathrm{H}-\mathrm{MoS}_{2}$ by thiolated surfactants: Applications in enhanced antibacterial activity. J. Am. Chem. Soc. 2018, 140, 12634-12644. [CrossRef] [PubMed]

5. Lu, J.Y.; Cai, L.; Zhang, N.; Qiu, B.C.; Chai, Y. Robust photoelectrochemical oxygen evolution with N, Fe-CoS nanorod arrays. ACS Appl. Mater. Interfaces. 2019, 11, 44214-44222. [CrossRef] [PubMed]

6. Wang, L.; Yue, Q.; Pei, C.; Fan, H.; Dai, J.; Huang, X.; Li, H.; Huang, W. Scrolling bilayer $W_{2} / \mathrm{MoS}_{2}$ heterostructures for high-performance photo-detection. Nano Res. 2020, 13, 959-966. [CrossRef]

7. Yu, M.; Kosinov, N.; van Haandel, L.; Kooyman, P.J.; Hensen, E.J.M. Investigation of the active phase in K-promoted $\mathrm{MoS}_{2}$ catalysts for methanethiol synthesis. ACS Catal. 2020, 10, 1838-1846. [CrossRef]

8. Wang, Y.; Mao, J.; Meng, X.G.; Yu, L.; Deng, D.H.; Bao, X.H. Catalysis with two-dimensional materials confining single atoms: Concept, design, and applications. Chem. Rev. 2019, 119, 1806-1854. [CrossRef] [PubMed]

9. Chia, X.Y.; Pumera, M. Layered transition metal dichalcogenide electrochemistry: Journey across the periodic table. Chem. Soc. Rev. 2018, 47, 5602-5613. [CrossRef]

10. Morrish, R.; Haak, T.; Wolden, C.A. Low-temperature Synthesis of n-type $\mathrm{WS}_{2}$ thin films via $\mathrm{H}_{2} \mathrm{~S}$ plasma sulfurization of $\mathrm{WO}_{3}$. Chem. Mater. 2014, 26, 3986-3992. [CrossRef] 
11. Liu, S.; Zeng, Y.; Zhang, M.; Xie, S.; Tong, Y.; Cheng, F.; Lu, X. Binder-free $\mathrm{WS}_{2}$ nanosheets with enhanced crystallinity as a stable negative electrode for flexible asymmetric supercapacitors. J. Mater. Chem. A 2017, 5, 21460-21466. [CrossRef]

12. Meng, L.; Hu, S.; Xu, C.; Wang, X.; Li, H.; Yan, X. Surface enhanced Raman effect on CVD growth of WS film. Chem. Phys. Lett. 2018, 707, 71-74. [CrossRef]

13. Kim, H.S.; Patel, M.; Kim, J.; Jeong, M.S. Growth of wafer-scale standing layers of $\mathrm{WS}_{2}$ for self-biased high-speed UV-Visible-NIR optoelectronic devices. ACS Appl. Mater. Interfaces 2018, 10, 3964-3974. [CrossRef] [PubMed]

14. Nguyen, T.N.; Patel, M.; Ban, D.K.; Kim, J. Vertically trigonal $\mathrm{WS}_{2}$ layer embedded heterostructure for enhanced ultraviolet-visible photodetector. J. Alloy Compd. 2018, 768, 143-149. [CrossRef]

15. Voiry, D.; Yamaguchi, H.; Li, J.; Silva, R.; Alive, D.C.; Fujita, T.; Chen, M.; Asefa, T.; Shenoy, V.B.; Eda, G.; et al. Enhanced catalytic activity in strained chemically exfoliated $\mathrm{WS}_{2}$ nanosheets for hydrogen evolution. Nat. Mater. 2013, 12, 850. [CrossRef]

16. Li, H.; Wu, H.; Yuan, S.; Qian, H. Synthesis and characterization of vertically standing $\mathrm{MoS}_{2}$ nanosheets. Sci. Rep. 2016, 6, 21171. [CrossRef]

17. Li, X.-L.; Ge, J.-P.; Li, Y.-D. Atmospheric pressure chemical vapor deposition: An alternative route to large-Scale $\mathrm{MoS}_{2}$ and $\mathrm{WS}_{2}$ inorganic fullerene-like nanostructures and nanoflowers. Chem. Eur. J. 2004, 10, 6163-6171. [CrossRef]

18. Fleischmann, M.; Hendra, P.J.; McQuillan, A.J. Raman Spectra of pyridine adsorbed at a silver electrode. Chem. Phys. Lett. 1974, 26, 163-166. [CrossRef]

19. Moskovits, M. Surface-Enhanced Spectroscopy. Rev. Mod. Phys. 1985, 57, 783-826. [CrossRef]

20. Li, J.F.; Huang, Y.F.; Ding, Y.; Yang, Z.L.; Li, S.B.; Zhou, X.S.; Fan, F.R.; Zhang, W.; Zhou, Z.Y.; Wu, D.Y.; et al. Shell-isolated nanoparticle-enhanced Raman spectroscopy. Nature 2010, 464, 392-395. [CrossRef]

21. Schlücker, S. Surface-enhanced Raman spectroscopy: Concepts and chemical applications. Angew. Chem. Int. Ed. 2014, 53, 4756-4795. [CrossRef] [PubMed]

22. Lombardi, J.R.; Birke, R.L. Theory of surface-enhanced Raman scattering in semiconductors. J. Chem. Phys. C 2014, 118, 11120-11130. [CrossRef]

23. Shin, H.Y.; Shim, E.L.; Choi, Y.J.; Park, J.H.; Yoon, S. Giant enhancement of the Raman response due to one-dimensional ZnO nanostructures. Nanoscale 2014, 6, 14622-14626. [CrossRef] [PubMed]

24. Alessandri, I.; Lombardi, J.R. Enhanced Raman scattering with dielectrics. Chem. Rev. 2016, 116, 14921-14981. [CrossRef] [PubMed]

25. Kannan, P.K.; Shankar, P.; Blackman, C.; Chung, C.H. Recent advances in 2D inorganic nanomaterials for SERS sensing. Adv. Mater. 2019, 31, 1803432. [CrossRef]

26. Muehlethaler, C.; Considine, C.R.; Menon, V.; Lin, W.C.; Lee, Y.H.; Lombardi, J.R. Ultrahigh Raman enhancement on monolayer $\mathrm{MoS}_{2}$. ACS Photonics 2016, 3, 1164-1169. [CrossRef]

27. Liu, Y.; Gao, Z.; Chen, M.; Tan, Y.; Chen, F. Enhanced Raman Scattering of CuPc Films on Imperfect WSe 2 Monolayer Correlated to Exciton and Charge-Transfer Resonances. Adv. Funct. Mater. 2018, 28, 1805710. [CrossRef]

28. Kim, S.; Yu, H.K.; Yoon, S.; Lee, N.S.; Kim, M.H. Growth of two-dimensional rhenium disulfide $\left(\operatorname{ReS}_{2}\right)$ nanosheets with a few layers at low temperature. CrystEngComm 2017, 19, 5341-5345. [CrossRef]

29. Mahler, B.; Hoepfner, V.; Liao, K.; Ozin, G.A. Colloidal synthesis of $1 \mathrm{~T}-\mathrm{WS}_{2}$ and $2 \mathrm{H}-\mathrm{WS}_{2}$ nanosheets: Applications of photocatalytic hydrogen evolution. J Am. Chem. Soc. 2014, 136, 14121-14127. [CrossRef]

30. Yang, J.; Voiry, D.; Ahn, S.J.; Kang, D.; Kim, A.Y.; Chhowalla, M.; Shin, H.S. Two-dimensional hybrid nanosheets of tungsten disulfide and reduced graphene oxide as catalysts for enhanced hydrogen evolution. Angew. Chem. Int. Ed. 2013, 125, 13996-13999. [CrossRef]

31. Kim, N.J.; Kim, J.; Park, J.B.; Kim, H.; Yi, G.C.; Yoon, S. Direct observation of quantum tunneling charge transfers between molecules and semiconductors for SERS. Nanoscale 2019, 11, 45-49. [CrossRef] [PubMed]

32. Kim, J.; Jang, Y.; Kim, N.J.; Kim, H.; Yi, G.C.; Shin, Y.; Kim, M.H.; Yoon, S. Study of Chemical Enhancement Mechanism in Non-plasmonic Surface Enhanced Raman Spectroscopy (SERS). Front. Chem. 2019, 7, 582. [CrossRef] [PubMed]

(C) 2020 by the authors. Licensee MDPI, Basel, Switzerland. This article is an open access article distributed under the terms and conditions of the Creative Commons Attribution (CC BY) license (http://creativecommons.org/licenses/by/4.0/). 